Pacific

Journal of

Mathematics

\title{
THE THURSTON NORM VIA NORMAL SURFACES
}

DARYL COOPER AND STEPHAN TILLMANN 


\title{
THE THURSTON NORM VIA NORMAL SURFACES
}

\author{
DARYl COOPER AND StePhan TILLMANN \\ To Bill Thurston on the occasion of his sixtieth birthday
}

\begin{abstract}
Given a triangulation of a closed, oriented, irreducible, atoroidal 3-manifold every oriented, incompressible surface may be isotoped into normal position relative to the triangulation. Such a normal oriented surface is then encoded by nonnegative integer weights, 14 for each 3-simplex, that describe how many copies of each oriented normal disc type there are. The Euler characteristic and homology class are both linear functions of the weights. There is a convex polytope in the space of weights, defined by linear equations given by the combinatorics of the triangulation, whose image under the homology map is the unit ball, $\mathscr{B}$, of the Thurston norm.

Applications of this approach include (1) an algorithm to compute $\mathscr{B}$ and hence the Thurston norm of any homology class, (2) an explicit exponential bound on the number of vertices of $\mathscr{B}$ in terms of the number of simplices in the triangulation, (3) an algorithm to determine the fibred faces of $\mathscr{B}$ and hence an algorithm to decide whether a 3-manifold fibres over the circle.
\end{abstract}

This work was inspired by a desire to understand the topological significance of the faces of the unit ball of the Thurston norm. The main result of this paper implies that the unit ball of the Thurston norm for a closed, orientable, irreducible, atoroidal 3-manifold is the projection under a linear map of a certain polyhedron in transversely oriented normal surface space. This polyhedron can be computed from a triangulation using linear algebra. In order to make this paper accessible to a wide audience we do not assume that the reader is familiar with the theory of normal surfaces. To keep the paper short we have considered only the case that $M$ is closed and orientable and have introduced the bare minimum of the theory of transversely oriented normal surfaces for this application. To facilitate a quick overview, we first state most definitions and results leading up to the main result (Theorem 5) as well as the applications, and fill the gaps at the end of the paper. A more general treatment is to be found in [Cooper and Tillmann $\geq 2008$ ], and references to normal surface theory can be found in [Jaco and Rubinstein 2003].

MSC2000: 57N10, 57M25.

Keywords: 3-manifold, Thurston norm, triangulation, normal surface.

This work was partially supported by NSF grant DMS-0405963. 
Transversely oriented normal surfaces. An arc $\alpha$ in a 2-simplex $\Delta$ is a normal arc if $\alpha \cap \partial \Delta=\partial \alpha$, and in addition the endpoints of $\alpha$ lie in the interior of distinct edges

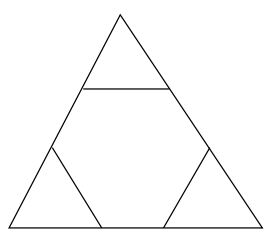
of $\Delta$. A transversely oriented normal arc $\left(\alpha, v_{\alpha}\right)$ is a normal arc $\alpha$ in $\Delta$ together with a transverse orientation $v_{\alpha}$ to $\alpha$ in $\Delta$. Two such arcs are equivalent if there is a homeomorphism of $\Delta$ to itself which preserves each edge of $\Delta$ and takes one arc with its transverse orientation to the other. There are 6 equivalence classes.

A disc $D$ in a 3-simplex $\tau$ is a normal disc if $D \cap \partial \tau=\partial D$ is a union of normal arcs, no two of which lie in the same face of $\tau$. The disc $D$ is called a triangle if the boundary consists of three normal arcs; otherwise the boundary has four nor-

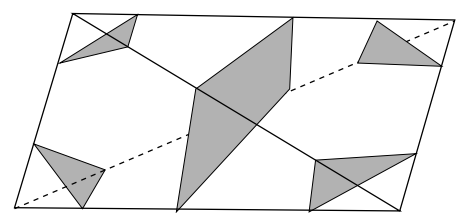
mal arcs and $D$ is called a quad. A transversely oriented normal disc $\left(D, v_{D}\right)$ is a normal disc $D$ together with a transverse orientation $v_{D}$. Two such discs are equivalent if there is a homeomorphism of $\tau$ to itself which preserves each face of $\tau$ and takes one disc with its transverse orientation to the other. There are 14 equivalence classes. The boundary of a transversely oriented normal disc is a collection of transversely oriented normal arcs, three for a triangle and four for a quad. Each transversely oriented normal arc is contained in the boundary of exactly one equivalence class of transversely oriented normal triangles and one of transversely oriented normal quads in $\tau$.

Unless stated otherwise, $M$ denotes a closed, oriented, irreducible 3-manifold and $\mathscr{T}$ a triangulation of $M$ with $t 3$-simplices. It is convenient to allow triangulations of $M$ which are more general than a simplicial triangulation. The interior of every simplex $\sigma$ in $\mathscr{T}$, of every dimension, is required to be embedded in $M$, but there may be self-identifications on the boundary of $\sigma$. A triangulation is 0-efficient if every normal 2-sphere is vertex linking, that is, it bounds a ball contained in a small neighbourhood of a vertex. Jaco and Rubinstein [2003] showed that if $M$ is not homeomorphic to $\mathbb{R} P^{3}$ or $L(3,1)$, then any minimal triangulation of $M$ is 0 -efficient. A minimal triangulation of $M$ has the property that no triangulation of $M$ contains fewer 3-simplices.

The transversely oriented normal disc space $N D^{v}(\mathscr{T})$ is the real vector space of dimension $14 t$ with a basis consisting of the equivalence classes of the transversely oriented normal discs in each 3-simplex of $\mathcal{T}$. A properly embedded surface $S \subset$ $M$ is a normal surface if the intersection of $S$ with every 3-simplex of $\mathscr{T}$ is a collection of pairwise disjoint normal discs. If the normal surface $S$ has a transverse orientation, $v_{S}$, then it determines a unique point $x^{v}\left(S, v_{S}\right) \in N D^{v}(\mathscr{T})$, where the coefficient of $\left(D, v_{D}\right)$ is the number of transversely oriented normal discs of that type in $S$. The following result is routine [Haken 1961; Jaco and Oertel 1984]: 
Proposition 1 (Incompressible is isotopic to normal). Every closed, incompressible surface in a closed, irreducible, triangulated 3-manifold is isotopic to a normal surface.

There is a linear subspace $N S^{v}(\mathscr{T}) \subset N D^{v}(\mathscr{T})$ defined by the matching equations. There is one matching equation for each equivalence class of transversely oriented normal arc $\left(\alpha, v_{\alpha}\right)$ in each 2-simplex $\Delta$ of $\mathscr{T}$. The two sides of $\Delta$ in $M$ are labelled + and - arbitrarily. The matching equation is

$$
t_{-}+q_{-}=t_{+}+q_{+} \text {. }
$$

Here $t_{-}$(respectively $q_{-}$) is the coefficient of the equivalence class of transversely oriented normal triangles (respectively quads) on the - side of $\Delta$ which contains $\left(\alpha, v_{\alpha}\right)$ in its boundary. Similarly for the + side. This equation expresses that there are the same number of transversely oriented normal discs containing $\left(\alpha, v_{\alpha}\right)$ in their boundary on either side of $\Delta$. It follows that if $\left(S, v_{S}\right)$ is a transversely oriented normal surface in $M$, then $x^{\nu}\left(S, v_{S}\right) \in N S^{\nu}(\mathcal{T})$.

An open normal $i$-cell in $M$ is the interior of a normal disc if $i=2$, the interior of a normal arc if $i=1$, and a point in the interior of a 1-simplex of $M$ if $i=0$. A branched immersed normal surface in $M$ is a pair $(S, f)$, where $S$ is a closed surface equipped with a cell decomposition and $f: S \rightarrow M$ satisfies

(1) if $\sigma$ is an open $i$-cell in $S$, then $f \mid \sigma$ is a homeomorphism onto an open normal $i$-cell in $M$, and

(2) for every open 1-cell $e$ in $S$ let $U$ be a small open disc neighborhood of $e$ in $S$ such that $e$ separates $U$ into two connected components $U_{ \pm}$, then $f\left(U_{ \pm}\right)$lie on locally opposite sides of the 2-simplex in $M$ which contains $f(e)$.

It follows that $f$ is locally injective except at the 0-cells. At each 0-cell $x$ in $S$ choose a small disc $D \subset S$ containing $x$. Let $e$ be the 1-cell in $\mathscr{T}$ containing $f(x)$ and $B$ a small ball in $M$ containing $f(x)$ such that $e \cap B$ is an arc. The branch index $\beta(f ; x)$ of $f$ at $x$ is the absolute value of

$$
f_{*}([\partial D]) \in H_{1}(B \backslash e) \cong \mathbb{Z} .
$$

This is just the local winding number of $f \mid \partial D$ around $e$.

As an example, suppose $F$ is an embedded normal surface in $M$ and $\pi: S \rightarrow F$ is a branched cover branched over a subset of the intersection of $F$ with the 1skeleton of $M$. Then $\pi$ composed with the inclusion of $F$ into $M$ is a branched immersion of $S$ into $M$. The cell decomposition of $S$ is just the lift of the cell decomposition of $F$ given by the normal discs and arcs that comprise $F$.

Proposition 2 (Branched immersions). Every nonzero point in $N S^{\nu}(\mathscr{T})$ with nonnegative integral coordinates is represented by a transversely oriented normal 
branched immersion of a closed orientable surface. Conversely, a transversely oriented normal branched immersion of a closed orientable surface, $\left(f, v_{f}\right): S \rightarrow M$, determines a unique point, $x^{\nu}\left(f, v_{f}\right)$, in $N S^{\nu}(\mathscr{T})$.

A convex polytope is the intersection of a finite number of closed affine halfspaces in Euclidean space. A point, $x$, in such a polytope is called a vertex if there is a codimension- 1 hyperplane whose intersection with the polytope equals $x$. It is easy to see that a compact, convex polytope is the convex hull of its vertices; see [Ziegler 1995]. The normal surface space is

$$
N S_{+}^{\nu}(\mathscr{T})=N S^{\nu}(\mathscr{T}) \cap[0, \infty)^{14 t} .
$$

It is a cone on the compact convex polytope $\mathscr{C}=N S_{+}^{v}(\mathscr{T}) \cap V$, where $V$ is the affine subspace consisting of all points whose coordinates sum to one. Whence $\mathscr{C}$ is the intersection of a simplex of dimension $14 t-1$ with a linear subspace and is therefore a compact convex polytope called the projective solution space. Given a transversely oriented normal surface, we would like to know its Euler characteristic as well as which homology class it represents. This information is given by the linear maps described in the following two results.

Lemma $3\left(\chi^{*}=\chi\right.$ for immersions). There is a linear map $\chi^{*}: N S^{\nu}(\mathscr{T}) \rightarrow \mathbb{R}$ with the property that if $\left(f, v_{f}\right): S \rightarrow M$ is a transversely oriented normal (unbranched) immersion, then $\chi^{*}\left(\chi^{v}\left(f, v_{f}\right)\right)=\chi(S)$.

The equality in the above lemma does not hold for branched immersions.

If $(S, f)$ is a branched immersed normal surface, and $S$ is oriented, then the orientation on $M$ determines an induced transverse orientation for the immersion $v_{f}$. Conversely, given a transversely oriented normal branched immersion of a closed surface $S$, the transverse orientation and the orientation on $M$ determine an orientation of $S$. This observation leads to:

Proposition 4 (Homology map). There is a surjective homomorphism

$$
h: N S^{\nu}(\mathscr{T}) \rightarrow H_{2}(M ; \mathbb{R}),
$$

called the homology map, with the following property: If $\left(f, v_{f}\right): S \rightarrow M$ is a transversely oriented normal branched immersion, then $f_{*}([S])=h\left(x^{v}\left(f, v_{f}\right)\right)$, where $S$ is given the induced orientation.

A point $x \in N S_{+}^{\nu}(\mathscr{T})$ is admissible if at most one quad type appears in each 3 -simplex (though both orientations are allowed). Two points $x, y \in N S_{+}^{\nu}(\mathscr{T})$ are compatible if $x+y$ is admissible.

Theorem 5 (Thurston norm via normal surfaces). Let $M$ be a closed, orientable, irreducible, atoroidal 3-manifold with simplicial or 0-efficient triangulation $\mathcal{T}$. Let 
$\mathscr{G}$ be the projective solution space and $B$ be the convex hull of the finite set of points $\left\{\frac{v}{\left|\chi^{*}(v)\right|}: v\right.$ is a vertex of $\mathscr{b}$ which is admissible and satisfies $\left.\chi^{*}(v)<0\right\}$.

If $\operatorname{dim} H_{2}(M)>0$, then $h(B)$ is the unit ball, $\mathscr{B}$, of the Thurston norm on $H_{2}(M ; \mathbb{R})$. In particular, $\mathscr{B}$ has at most $2^{14 t}$ vertices.

Algorithm 6 (Fibred faces). Let $M$ be a closed, orientable, irreducible, atoroidal 3-manifold. The following is an algorithm to determine the fibred faces of $\mathscr{B}$ and hence to determine whether $M$ fibres over the circle:

0 . We may assume that $M$ is given via a (simplicial or 0-efficient) triangulation.

1. If $\operatorname{dim} H_{2}(M)=0$, then $M$ is not fibred. Otherwise compute the unit norm ball of the Thurston norm using the above theorem.

2. Determine the top-dimensional faces.

3. For each top-dimensional face $\mathscr{F}$ of $\mathscr{B}$ :

a Let $\hat{\mathscr{F}}$ be the barycentre of $\mathscr{F}$ and let $\alpha \in H_{2}(M ; \mathbb{Z})$ be the smallest integral multiple of $\hat{\mathscr{F}}$.

b Find an embedded, norm minimising, transversely oriented normal surface $S$ without sphere or torus components representing $\alpha$.

c Use Haken's algorithm to check whether $\overline{M \backslash S}$ is homeomorphic to $S \times[0,1]$; see [Matveev 2003].

Then $\mathscr{F}$ is a fibred face if and only if Step 3.c yields an affirmative answer. Moreover, $M$ fibres over the circle if and only if at least one face is fibred.

This paper gives a self-contained proof of Theorem 5 for a 0 -efficient triangulation. In the case of a simplicial triangulation, work by Tollefson and Wang [1996] is used to prove Theorem 5. An alternative approach to construct $\mathscr{B}$ is given in [Tollefson and Wang 1996, Algorithm 5.9], and used by Schleimer [2001] to obtain an algorithm to determine whether a 3-manifold fibres over the circle. The algorithm to compute $\mathscr{B}$ given here is practical in the sense that the authors hope to implement it on a computer.

\section{Proofs, neat position and algebraically aspherical solutions.}

Proof of Proposition 2. A nonzero point in $N S^{\nu}(\mathscr{T})$ with nonnegative integral coordinates determines an abstract collection of normal discs. Since the integers satisfy the matching equations, we may identify the edges of these discs in pairs to produce a closed surface $S$ with a cell decomposition such that there is a map of $S$ into $M$ mapping each 2-cell in $S$ to a normal disc in $M$. This map is an immersion on the complement of the set, $V$, of vertices of $S$, and thus a branched immersion. The transverse orientations on the discs match along edges, so this map extends 
to a map of $S \times[-1,1]$ into $M$ which is an immersion on the complement of $V \times[-1,1]$. The map of $S$ therefore has a transverse orientation and, since $M$ is orientable, it follows that $S$ is orientable. The converse direction is obvious.

Proof of Lemma 3. The degree of a 1-simplex $e$ in $\mathscr{T}$ is the number of 3-simplices in $\mathscr{T}$ which contain $e$ counted with multiplicity. This means that if a 3-simplex $\tau$ has $n$ of its edges identified with $e$ then $\tau$ contributes $n$ to this count. We define $\chi^{*}$ on each transversely oriented normal disc $\left(D, v_{D}\right)$ as follows, and then extend linearly. For each vertex $x \in \partial D$ let $\delta(x)$ denote the degree of the 1-simplex in $\mathscr{T}$ that contains $x$. Then define

$$
\chi^{*}\left(D, v_{D}\right)=1-\frac{\#(\partial D)}{2}+\sum_{x \in \partial D} \frac{1}{\delta(x)},
$$

where $\#(\partial D)$ is the number of sides of $\partial D$ (three for a triangle and four for a quad). A normal immersion of a surface $S$ gives it a cell decomposition into normal triangles and quads. Every 1-cell appears in two 2-cells, and every 0-cell $x$ appears in $\delta(x)$ 2-cells. Summing the formula then gives the usual formula for the Euler characteristic for $S$ using this cell decomposition.

Proof of Proposition 4. Denote by $C_{2}(M)$ the 2-dimensional singular chain group of $M$ with real coefficients $\mathbb{R}$. A homomorphism $\bar{h}: N D^{v}(\mathscr{T}) \rightarrow C_{2}(M)$ is first constructed. Suppose $\left(D, v_{D}\right)$ is a transversely oriented normal disc in some 3simplex of $\mathscr{T}$. The orientation of $M$ and the transverse orientation $v_{D}$ determine an orientation $\mu_{D}$ of $D$. If $D$ is a normal triangle then $\left(D, \mu_{D}\right)$ may be regarded as a singular 2-simplex. If $D$ is a normal quad, we add a diagonal to subdivide $D$ into two triangles and obtain the sum of two singular 2-simplices. This defines $\bar{h}$ on a basis and we extend linearly. It is easy to see that if $x \in N S^{\nu}(\mathscr{T})$ then $\bar{h}(x)$ is a singular 2-cycle. See [Cooper and Tillmann $\geq 2008$ ] for details. We define $h(x)=[\bar{h}(x)]$. It is also easy to see that if $\left(f, v_{f}\right): S \rightarrow M$ is a transversely oriented branched normal immersion then $f_{*}([S])=h\left(x^{v}\left(f, v_{f}\right)\right)$. The map $h$ is surjective since every homology class is represented by an embedded, closed, oriented surface which may be isotoped to be normal.

We obtain the theory of normal surfaces if we forget transverse orientations. Thus $N D(\mathscr{T})$ is the vector space of dimension $7 t$ with basis the equivalence classes of normal discs in each 3 -simplex of $\mathscr{T}$. There is one unoriented matching equation for each equivalence class of normal arc in each 2 -simplex in $\mathscr{T}$. The solutions of the matching equations give a linear subspace $N S(\mathscr{T}) \subset N D(\mathscr{T})$, and

$$
N S_{+}(\mathscr{T})=N S(\mathscr{T}) \cap[0, \infty)^{7 t} .
$$

A point in $N S_{+}(\mathscr{T})$ is admissible if in each 3-simplex the coefficient of at most one quad type is nonzero. 
We now describe the geometric sum of two embedded normal surfaces. This idea goes back to Haken [1961]. Suppose $D, D^{\prime}$ are two normal discs in a 3-simplex $\tau$ which intersect transversely in an $\operatorname{arc} \alpha$. After cutting $D$ and $D^{\prime}$ along $\alpha$ there are two possible ways to cross join to obtain two disjoint discs. There is exactly one way which yields disjoint normal discs unless $D$ and $D^{\prime}$ are quads of different types. In that case there is no cross join which gives disjoint normal discs. The direction of the cross join is determined by the way the normal arcs intersect in the boundary. Now suppose $S$ and $F$ are two normal surfaces. We may isotope them so they intersect transversely and, for every 3-simplex $\tau \in \mathscr{T}$, the intersection of a normal disc in $S \cap \tau$ with one in $F \cap \tau$ is either empty or a single arc. If $S$ and $F$ are compatible, that is, the sum of their normal coordinates is admissible, then there is a unique way to cut and cross join $S$ and $F$ along $S \cap F$ to obtain a new (possibly not connected) normal surface denoted $S+F$.

The pair $(S, F)$ is disc reduced if no curve of intersection in $S \cap F$ bounds a disc in both $S$ and $F$. An innermost discs argument shows that $S$ and $F$ can be replaced by normal surfaces $S^{\prime}$ and $F^{\prime}$ homeomorphic to (but possibly not normally isotopic to) $S$ and $F$ respectively such that $\left(S^{\prime}, F^{\prime}\right)$ is disc reduced and $S^{\prime}+F^{\prime}$ is normally isotopic to $S+F$. An ambient isotopy of $M$ is a normal isotopy if it preserves every cell in the triangulation $\mathscr{T}$. The following is well known (see [Tollefson 1995]):

Proposition 7 (Admissible implies unique embedded normal surface). Suppose $M$ is a closed, triangulated 3-manifold. There is a one-to-one correspondence between admissible nonnegative integral points in NS(T) and normal isotopy classes of embedded normal surfaces in M. Furthermore, addition of compatible points corresponds to geometric sum of compatible normal surfaces.

Proof. First assume that every simplex in $\mathscr{T}$ is embedded. A point $x \in N S_{+}(\mathscr{T})$ determines a collection of normal discs in each 3 -simplex of $\mathscr{T}$. These in turn determine a number of distinct points on each 1-simplex of $\mathscr{T}$. Up to normal isotopy, the position of these points on each 1-simplex is unique. These points determine a collection of normal arcs in each 2-simplex of $\mathscr{T}$. Up to normal isotopy there is a unique way to embed these arcs in each 2-simplex. Thus, up to normal isotopy, $x$ determines a unique set of disjoint simple closed curves, $C(x, \tau)$, in the boundary of each 3-simplex $\tau \in \mathscr{T}$. Up to normal isotopy there is a unique set of disjoint discs in $\tau$ with boundary $C(x, \tau)$. These discs are normal discs if and only if each simple closed curve in $C(x, \tau)$ meets each face of $\tau$ at most once. This happens if and only if $x$ is admissible. If $x$ is admissible these normal discs are identified along their boundaries to produce a normal surface $S$ which is thus unique up to normal isotopy. In the general case that $\mathcal{T}$ contains simplices which are not embedded, the above argument is modified by lifting back the construction from $M$ to the 3simplices of the triangulation. 
The proof of Proposition 7 relies on the fact that up to normal isotopy a family of disjoint normal arcs in a 2-simplex, $\Delta$, is determined by the number of endpoints of those arcs on each edge of $\Delta$. In the transversely oriented setting there are two types of points on each edge, distinguished by transverse orientation. We no longer have a unique way, up to isotopy, to arrange these points along each edge; for example we can distinguish the linear orderings ++- from +-+ . Instead we make a natural choice called neat position: on each edge we put all the + points at one end and all the - points at the other end. This gives a way to position an admissible family of transversely oriented normal discs in a 3-simplex based only on the number of each type of transversely oriented points on each edge of the 3-simplex. We show this leads to an (unbranched) immersion.

However, if we start with an embedded transversely oriented normal surface, $F$, and decompose it into transversely oriented normal discs (with disjoint interiors), the + and - points we obtain may alternate along an edge. The neat position construction described below reorders these points and changes the positions of the normal discs comprising $F$. The discs might now intersect. The resulting neat position surface, $F^{\prime}$, obtained by identifying the boundaries of the neatly positioned discs is not embedded, even though it is made up of the same number of each type of transversely oriented normal discs as $F$. The discs are glued differently.

Proposition 8 (Admissible implies immersed transversely oriented normal). If $M$ is a closed, orientable, triangulated 3-manifold, then every admissible nonnegative integral point in $N S^{\nu}(\mathscr{T})$ is the coordinate of a transversely oriented (unbranched) normal immersion of a closed oriented surface.

Proof. A nonnegative admissible integral point $x^{\nu} \in N S^{\nu}(\mathscr{T})$ determines a collection of transversely oriented normal discs in each 3-simplex. We embed each of these discs in the simplex in a canonical neat position defined below. In each 3simplex the boundary arcs of different discs may intersect but only in the interior of 2 -simplices in $\mathcal{T}$, and canonical means that the normal discs in adjacent 3-simplices have boundary arcs that match pairwise with matching transverse orientations to give a transversely oriented immersed normal surface realising $x^{\nu}$.

First we define neat position. Suppose $\left(\alpha, v_{\alpha}\right)$ is a transversely oriented normal arc in a 2-simplex $\Delta$. The transverse orientation is thought of as a function on the components of $\Delta \backslash \alpha$ which sends one component to +1 and the other to -1 . Denote the former by $C_{+}$and let $\sigma=$ $\sigma\left(\alpha, v_{\alpha}\right)$ denote the maximal subsimplex of $\Delta$ contained

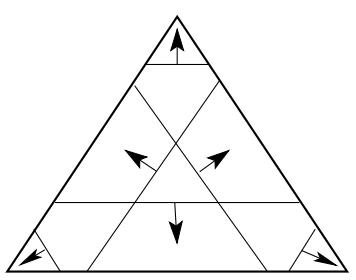
in $C_{+}$. There are 6 such simplices and they are in bijective correspondence with the equivalence classes of $\left(\alpha, v_{\alpha}\right)$. The arc is called short if $\sigma$ is a 0 -simplex. Otherwise $\sigma$ is a 1-simplex and the arc is called long. The standard 2-simplex 
means an equilateral triangle in the Euclidean plane with each side of length 1. A family of transversely oriented normal arcs in $\Delta$ is in neat position if there is an identification of $\Delta$ with the standard 2-simplex such that the arcs in the family satisfy:

- every arc is straight,

- every point on each arc $\left(\alpha, v_{\alpha}\right)$ in the family is within distance $\frac{1}{10}$ of $\sigma\left(\alpha, v_{\alpha}\right)$,

- arcs of the same unoriented type are pairwise disjoint, and

- every short arc is disjoint from every other arc.

It follows that the short arcs are contained in small neighborhoods of the vertices of $\Delta$ which are disjoint from the long arcs, and each long arc is very close to some edge of $\Delta$, and that two arcs intersect if and only if they are both long and of different unoriented types. In this case they intersect once transversely, and the intersection point is in the interior of $\Delta$. Given specified numbers of each type of transversely oriented normal arc, it is clear that, up to normal isotopy of $\Delta$ and permutation of the arcs, the configuration of the arcs in $\Delta$ is unique.

Suppose $\left(D, v_{D}\right)$ is a transversely oriented normal disc in a 3-simplex $\tau$. As above, a component of $\tau \backslash D$ is labelled $C_{+}$using $v_{D}$. Let $\sigma\left(D, v_{D}\right)$ denote the maximal subsimplex of $\tau$ contained in $C_{+}$. There are 14 such simplices and they are in bijective correspondence with the equivalence classes of transversely oriented normal discs. A transversely oriented normal triangle, $\left(D, v_{D}\right)$, is called small if $\sigma=\sigma\left(D, v_{D}\right)$ is a 0 -simplex and otherwise it is called large (in which case $\sigma$ is a 2-simplex).

A family of transversely oriented normal discs in a 3-simplex $\tau$ is in neat position if

- the family is in general position, and

- the intersection of the family with each 2 -simplex $\Delta \subset \partial \tau$ is a family of transversely oriented arcs in neat position.

Suppose we are given a collection of transversely oriented normal discs in a 3 -simplex $\tau$ which is admissible: only one quad type appears but both transverse orientations are allowed. We show below that these discs may be placed in neat position. Though we do not need this fact, it is easy to see that two quads of different unoriented types can never be in neat position.

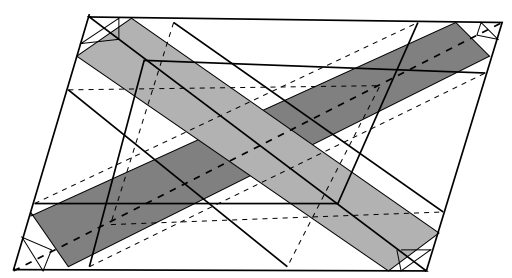

It suffices to show that it is possible to place in neat position one copy of each transversely oriented normal triangle in $\tau$ together with two normal quads which differ only in transverse orientation. Having done this, one may take closely spaced parallel copies of these discs. The figure 
shows how to place the discs. First place the large triangles in neat position. Next place each transversely oriented quad in a small neighborhood of the 1-simplex of $\sigma$ it determines, so that it is disjoint from the large triangles it can be made disjoint from. Finally place the small triangles in small neighbourhoods of the vertices disjoint form all other discs. This completes the claim.

We now describe canonical neat position for admissible solutions. To do this we put compatible Euclidean structures on the 2-simplices as follows. First we choose an identification of each 1-simplex in $\mathscr{T}$ with the unit interval. This puts a path metric on each 1-simplex. Next we choose an identification of every 2simplex, $\Delta$, in $\mathscr{T}$ with the standard 2 -simplex so that the induced metric on each 1-simplex, $\sigma$, in the boundary of $\Delta$ agrees with that given by the identification of $\sigma$ with the unit interval.

A vector $x^{v}$ in $N S_{+}(\mathscr{T})$ determines, for each 1-simplex $e$ of $\mathscr{T}$, a finite number of transversely oriented points on $e$. We first position these points on $e$ in neat position which just means within distance $\frac{1}{10}$ of the end of $e$ determined by the transverse orientation on that point, and equally spaced apart. The vector $x^{v}$ also determines a family of transversely oriented normal arcs types in each 2-simplex of $\mathcal{T}$. By the previous discussion, there is a unique way to place these normal arcs in neat position with endpoints the chosen neatly positioned points. Since $x^{\nu}$ is admissible, there is then a unique way to place the transversely oriented normal disc types determined by $x^{v}$ in each 3 -simplex of $\mathscr{T}$ into neat position so the boundary is the set of neatly positioned arcs. This is the canonical neat position.

Since $M$ is orientable and the immersed surface constructed above is transversely orientable it is also orientable.

An orientable surface $S$ is aspherical if no connected component of $S$ is a sphere. We wish to have an algebraic criterion for deciding whether a transversely oriented normal surface is aspherical depending only on its transversely oriented normal coordinates. There is a partial order $\geq$ on $N D^{v}(\mathscr{T})$, where $x \geq y$ if and only if every coordinate of $x$ is greater than or equal to the corresponding coordinate of $y$.

Definition. A point $x \in N S_{+}^{v}(\mathscr{T})$ is algebraically aspherical if for all $y \in N S_{+}^{v}(\mathscr{T})$ such that $x \geq y$, we have $\chi^{*}(y) \leq 0$.

Lemma 9 (Properties of aspherical elements). The following properties follow directly from the definition:

(1) If $x \in N S_{+}^{v}(\mathscr{T})$ is algebraically aspherical, $\chi^{*}(x) \leq 0$.

(2) If $x=\sum x_{i}$ is algebraically aspherical and $x_{i} \in N S_{+}^{\nu}(\mathscr{T})$ for each $i$, then each $x_{i}$ is algebraically aspherical.

(3) If $x \in N S_{+}^{\nu}(\mathscr{T})$ is algebraically aspherical, so is $\alpha x$ for any $\alpha>0$. 
Proposition 10 (embedded aspherical in 0 -efficient $\Rightarrow$ algebraically aspherical). Let $M$ be a closed, oriented 3-manifold with 0-efficient triangulation $\mathscr{T}$. If $\left(F, v_{F}\right)$ is an embedded transversely oriented normal surface in $M$ and no component of $F$ is a sphere, then $x^{\nu}\left(F, v_{F}\right)$ is admissible and algebraically aspherical.

Proof. Since $F$ is embedded $x^{\nu}=x^{\nu}\left(F, v_{F}\right)$ is admissible. Forgetting transverse orientations gives a linear map $\varphi: N S^{\nu}(\mathscr{T}) \rightarrow N S(\mathscr{T})$, and we write $x=\varphi\left(x^{\nu}\right)$. The topology of the embedded normal surface $F$ is determined (up to normal isotopy) by $x$. If $x^{\nu}$ is not algebraically aspherical, we will show that there is an integer $n>0$ such that $n x>y$, where $y=x(S)$ for some embedded normal sphere $S$. Then

$$
n x=(n x-y)+y,
$$

where $n x,(n x-y), y \in N S_{+}(\mathscr{T})$ are compatible admissible nonnegative integral solutions to the unoriented matching equations. By Proposition 7 for each admissible $z \in N S_{+}(\mathscr{T})$ there is an embedded normal surface $F(z)$ in $M$ with normal coordinates $z$, and this surface is unique up to normal isotopy. Thus $F(y)$ is normally isotopic to $S$. Since $\mathscr{T}$ is 0 -efficient $S$ is a vertex linking sphere and so may be isotoped into a small neighborhood of that vertex disjoint from $F(n x-y)$. Let $n \cdot F$ denote an embedded normal surface consisting of $n$ parallel disjoint copies of the normal surface $F$. The uniqueness part of Proposition 7 implies $F(n x)=n \cdot F$. The above equation implies $n \cdot F$ is normal-isotopic to the geometric sum of $F(n x-x(S))$ and $S$. Since these surfaces are disjoint, their geometric sum equals their disjoint union. Thus $n \cdot F$ contains a sphere normally isotopic to $S$, which is a contradiction.

It remains to prove that if $x^{\nu}$ is not algebraically aspherical, then $S$ exists. If $x^{\nu}$ is not algebraically aspherical, then there is $a^{v} \in N S_{+}^{v}(\mathscr{T})$ with $x^{v} \geq a^{v}$ and $\chi^{*}\left(a^{v}\right)>0$. We may assume $a^{v}$ has nonnegative rational coordinates. Then there is an integer $n>0$ so that $b^{v}=n a^{\nu}$ has integer coordinates and $n x^{\nu} \geq b^{\nu}$. Now $\chi^{*}\left(b^{v}\right)=n \chi^{*}\left(a^{v}\right)>0$. Since $x^{\nu}$ is admissible, $b^{v}$ is admissible and hence

$$
b=\varphi\left(b^{v}\right) \in N S_{+}(\mathscr{T})
$$

is admissible. The map $\chi^{*}$ factors through a linear map $\chi_{u n}^{*}: N D(\mathscr{T}) \rightarrow \mathbb{R}$ with the property that if $A$ is an embedded normal surface, then $\chi_{u n}^{*}(x(A))=\chi(A)$. So $\chi^{*}\left(b^{v}\right)=\chi_{u n}^{*}\left(\varphi\left(b^{v}\right)\right)$ and since $F(b)$ is an embedded normal surface,

$$
\chi(F(b))=\chi_{u n}^{*}(b)=\chi^{*}\left(b^{v}\right) .
$$

Hence $\chi(F(b))>0$. Thus $F(b)$ contains a component $S$ with $\chi(S)>0$. Since $S \subset F(b)$ are normal surfaces it follows that $b \geq x(S)$. If $S=\mathbb{R} P^{2}$ then, since $M$ is orientable, a small regular neighborhood of $S$ is bounded by a 2-sphere $S^{\prime}$, and $x\left(S^{\prime}\right)=2 x(S)$. Since $\mathcal{T}$ is 0 -efficient, $S^{\prime}$ is a vertex linking sphere. But this 
implies that $x(S)$ is not an integral solution, giving a contradiction. Thus $S$ is a sphere and $n x \geq b \geq x(S)$.

If $S$ is a closed surface, then $-\chi_{-}(S)$ is the sum of the Euler characteristics over all connected components of $S$ with negative Euler characteristic (see [Thurston 1986]). If $c \in H_{2}(M)$, then the Thurston norm of $c$ is

$$
\|c\|=\inf _{S:[S]=c} \chi_{-}(S) .
$$

If $M$ is irreducible, discarding sphere components from $S$ does not change [S] or $\chi_{-}(S)$ and so the infimum can be taken over surfaces containing no spheres.

A transversely oriented normal surface $\left(F, v_{F}\right)$ in $M$ is called a taut normal surface if it is aspherical and $\chi(F)=-\left\|h\left(x^{v}\left(F, v_{F}\right)\right)\right\|$, and it is least weight taut if $F$ minimises $\left|F \cap \mathscr{T}^{(1)}\right|$ over all taut normal surfaces representing $h\left(x^{v}\left(F, v_{F}\right)\right)$. The following is a reformulation of (part of) [Tollefson and Wang 1996, Lemma 3.2].

Lemma 11. Let $M$ be a closed, oriented 3-manifold with simplicial triangulation T. Let $\left(F, v_{F}\right)$ be least weight taut. Let $G, H$ be orientable normal surfaces such that

$$
m x(F)=x(G)+x(H)
$$

for some positive integer $m$ and the pair $(G, H)$ is disc reduced. Then the transverse orientation of $F$ induces unique transverse orientations $v_{G}$ and $v_{H}$ on $G$ and $H$ respectively such that $m x^{\nu}\left(F, v_{F}\right)=x^{\nu}\left(G, v_{G}\right)+x^{v}\left(H, v_{H}\right)$ and $\left(G, v_{G}\right)$ and $\left(H, v_{H}\right)$ are least weight taut.

Proposition 12 (least weight taut $\Rightarrow$ algebraically aspherical). Let $M$ be a closed, oriented, irreducible 3-manifold with simplicial triangulation $\mathscr{T}$. If $\left(F, v_{F}\right)$ is a least weight taut normal surface in $M$, then $x^{\nu}\left(F, v_{F}\right)$ is admissible and algebraically aspherical.

The proof of Proposition 10 is modified as follows.

Proof. First note that if $(F(n x-y), F(y))$ is not disc reduced, then there is $y^{\prime}$ such that $\left(F\left(n x-y^{\prime}\right), F\left(y^{\prime}\right)\right)$ is disc reduced and $\chi\left(F\left(y^{\prime}\right)\right)=\chi(F(y))=2$. Lemma 11 implies that $F\left(y^{\prime}\right)$ can be transversely oriented to give a least weight taut normal surface. Since $F\left(y^{\prime}\right) \neq \varnothing$, it follows that a sphere represents a nontrivial homology class contradicting the assumption that $M$ is irreducible.

Proof of Theorem 5. We claim that $\|h(y)\| \leq\left|\chi^{*}(y)\right|$ for all $y \in \operatorname{cone}(B)$, the cone of $B$ over the origin. It then follows that $h(B) \subseteq \mathscr{B}$ since $B=\operatorname{cone}(B) \cap$ $\left(\chi^{*}\right)^{-1}(-1)$. First we prove the claim in the special case when $y=v$ is a vertex of $B$. Since the inequalities defining $\mathscr{b}$ have integral coefficients, $v$ has rational coordinates. Let $t>0$ be minimal subject to that $x=t \cdot v$ is an integral point. Since $B \subset N S_{+}^{v}(\mathscr{T}), x$ is nonnegative. Since $v$ is admissible, $x$ is admissible. 
By Proposition 8 there is a transversely oriented (unbranched) normal immersion $\left(f, v_{f}\right): S \rightarrow M$ such that $x^{v}\left(f, v_{f}\right)=x$, and Proposition 4 gives $h(x)=f_{*}([S]) \in$ $H_{2}(M)$. Since $S$ is (unbranched) immersed, Lemma 3 yields $\chi^{*}(x)=\chi(S)$. If $S$ is not connected, then $x$ is the sum over all components $S_{i} \subset S$ of $x\left(S_{i}\right) \in \operatorname{cone}(\mathscr{C})$. Since $v$ is an extreme point of $\mathscr{C}$, it is not a nontrivial convex combination of points in $\mathscr{C}$. However,

$$
x^{\nu}\left(f, v_{s}\right)=\sum_{i} x^{\nu}\left(f\left|S_{i}, v_{f}\right| S_{i}\right) .
$$

Thus $x^{\nu}\left(f, v_{s}\right)$ is a multiple of $x^{\nu}\left(f\left|S_{i}, v_{f}\right| S_{i}\right)$ for every component $S_{i}$ of $S$. By minimality of $t$ it follows that $S$ is connected. Now $\chi^{*}(x)=t \chi^{*}(v)<0$ and thus $S$ is not a sphere, hence $-\chi_{-}(S)=\chi(S)=\chi^{*}(x)$. Gabai [1983] showed that the embedded norm equals the singular norm, and so

$$
\|h(x)\| \leq \chi_{-}(S)=|\chi(S)|=\left|\chi^{*}(x)\right| .
$$

This proves the claim in the special case.

To prove the claim in the general case, assume $y=\sum \lambda_{i} v_{i}$ with $\lambda_{i} \geq 0$, where each $v_{i}$ is a vertex of $B$. Then $h(y)=\sum \lambda_{i} h\left(v_{i}\right)$ so $\|h(y)\| \leq \sum \lambda_{i}\left\|h\left(v_{i}\right)\right\|$. Now $\chi^{*}(y)=\chi^{*}\left(\sum \lambda_{i} v_{i}\right)=\sum \lambda_{i} \chi^{*}\left(v_{i}\right)$. Since $\chi^{*}\left(v_{i}\right)<0$ we get

$$
\left|\chi^{*}(y)\right|=\sum \lambda_{i}\left|\chi^{*}\left(v_{i}\right)\right| .
$$

By the special case, $\left\|h\left(v_{i}\right)\right\| \leq\left|\chi^{*}\left(v_{i}\right)\right|$, and it follows that $\|h(y)\| \leq\left|\chi^{*}(y)\right|$, proving the claim.

To prove the reverse containment, a rational point in $\beta \in \partial \mathscr{B}$ can be expressed as $\beta=[S] /|\chi(S)|$ for some norm-minimising, transversely oriented embedded surface $\left(S, v_{S}\right)$ no component of which is a sphere or a torus. By Proposition $1 S$ can be isotoped into normal position. The ray through $p=x^{v}\left(S, v_{S}\right)$ intersects $\mathscr{C}$ at a point $x$. Since $p$ is admissible, $x$ is admissible. Lemma 3 gives $\left|\chi^{*}(p)\right|=|\chi(S)|=\|[S]\|$. Since $S$ is embedded and aspherical, Proposition 10 implies that $p$ is algebraically aspherical if $\mathscr{T}$ is 0-efficient. Otherwise, it follows from [Tollefson and Wang 1996, Lemma 2.1], that $\left(S, v_{S}\right)$ may be chosen to be least weight taut in which case Proposition 12 implies that $p$ is algebraically aspherical. Hence $x$ is algebraically aspherical by Lemma 9(3). We can express $x$ as a convex linear combination of some of the vertices of $\mathscr{C}$. Then $x=\sum t_{i} \cdot x_{i}$, where each $x_{i}$ is a vertex of $\mathscr{C}$ and $0<t_{i} \leq 1$. Parts (2) and (3) of Lemma 9 imply that each $x_{i}$ is algebraically aspherical, and therefore by Lemma $9(1) \chi^{*}\left(x_{i}\right) \leq 0$. Moreover, $x_{i} \leq x$; therefore $x_{i}$ is admissible. If $\chi^{*}\left(x_{i}\right)=0$, then the smallest integral multiple of $x_{i}$ is the normal coordinate of a connected, immersed surface of zero Euler characteristic, giving $h\left(x_{i}\right)=0$ since $M$ is atoroidal.

Let $\hat{x}=\sum t_{i} \cdot \hat{x}_{i}$, where $\hat{x}_{i}=x_{i}$ if $\chi^{*}\left(x_{i}\right)<0$ and $\hat{x}_{i}=0$ otherwise. Hence $h(\hat{x})=h(x), \chi^{*}(x)=\chi^{*}(\hat{x})$ and each nonzero $\hat{x}_{i}$ is in cone $(B)$. Since cone $(B)$ is 
convex, $\hat{x} \in \operatorname{cone}(B)$. It follows that

$$
\hat{x} /\left|\chi^{*}(\hat{x})\right| \in \operatorname{cone}(B) \cap\left(\chi^{*}\right)^{-1}(-1)=B .
$$

Now $[S]=h(p)$ and

$$
h(\hat{x}) /\left|\chi^{*}(\hat{x})\right|=h(x) /\left|\chi^{*}(x)\right|=h(p) /\left|\chi^{*}(p)\right|=[S] /|\chi(S)|=\beta \in \partial \mathscr{B} .
$$

Hence $h(B)$ contains all the rational points in $\partial \mathscr{B}$. The set of rational points in $\partial \mathscr{B}$ is dense because the Thurston norm takes integral values on integral points. Thus $\partial \mathscr{B} \subseteq h(B)$. Since $h$ is linear and $B$ is convex, $\mathscr{B} \subseteq h(B)$.

Each vertex of $\mathscr{C}=\Delta^{14 t-1} \cap N S^{\nu}(\mathscr{T})$ lies in a unique minimal subsimplex of $\Delta$. The subsimplices of $\Delta^{14 t-1}=V \cap[0, \infty)^{14 t}$ correspond to coordinate subspaces of $\mathbb{R}^{14 t}$. Thus a vertex of $\partial \mathscr{C}$ is uniquely determined by which normal coordinates are zero. There are $14 t$ coordinates, so $\mathscr{C}$ has at most $2^{14 t}$ vertices. Every vertex of the unit ball of the Thurston norm ball, $\mathscr{B}$, is the image of a point on a ray through a vertex of $\mathscr{C}$. This gives the claimed bound on the number of vertices of $\mathscr{B}$.

Proof of Algorithm 6. Only part 3.b needs to be explained. Let $\mathscr{C}_{\leq}$be the convex hull of the finite set of all vertices $v$ of $\mathscr{C}$ which are admissible and satisfy $\chi^{*}(v) \leq 0$. Define

$$
Q=\operatorname{cone}\left(\mathscr{C}_{\leq}\right) \cap\{x: h(x)=\alpha\} \cap\left\{x: \chi^{*}(x)=-\|\alpha\|\right\} .
$$

The total weight of a point in $Q$ is the sum of its coordinates. Given an integer $w>0$ let $\mathscr{P}(w)$ be the finite (possibly empty) set consisting of all admissible integral points in $Q$ of total weight at most $w$. The algorithm to construct $S$ is to increase $w$ until one of the points in $\mathscr{P}(w)$ is found, by the following procedure, to be the coordinate of an embedded transversely oriented normal surface without sphere or torus components. Given $x^{\nu} \in \mathscr{P}(w)$, construct the unique embedded normal surface $F$ with unoriented normal coordinate $x=\varphi\left(x^{\nu}\right)$. Discard $x^{\nu}$ if some component of $F$ is a torus, a sphere or 1-sided. Otherwise check whether the components of $F$ can be transversely oriented to yield an embedded transversely oriented normal surface with coordinate $x$.

We now prove this algorithm terminates. Let $F$ be a norm-minimising oriented surface without sphere or torus components and with $[F]=\alpha \in H_{2}(M)$, so $\|\alpha\|=$ $-\chi(F)$. By Proposition 1 we may assume $F$ is a transversely oriented normal surface and set $p=x^{v}\left(F, v_{F}\right)$. Then $p \in Q$ and $p \in \mathscr{P}(w)$ for $w$ sufficiently large. Hence the algorithm will construct $F$ or a surface with the same properties of lower weight.

Remark 13. If $M$ is a closed, oriented 3-manifold, let $\mathscr{C}_{\leq}$be the convex hull of the finite set of all vertices $v$ of $\mathscr{C}$ which are admissible and satisfy $\chi^{*}(v) \leq 0$, and let $B_{\leq}=\left\{x \in \operatorname{cone}\left(\mathscr{C}_{\leq}\right): \chi^{*}(x)=-1\right\}$. Then $h\left(B_{\leq}\right)$is the unit ball of the Thurston norm - this is a noncompact polytope if $M$ is not atoroidal. 


\section{Acknowledgment}

The authors thank Andrew Casson and Bus Jaco for helpful comments on this project.

\section{References}

[Cooper and Tillmann $\geq 2008$ ] D. Cooper and S. Tillmann, "Transversely oriented normal surfaces", in preparation.

[Gabai 1983] D. Gabai, "Foliations and the topology of 3-manifolds", J. Differential Geom. 18:3 (1983), 445-503. MR 86a:57009 Zbl 0533.57013

[Haken 1961] W. Haken, “Theorie der Normalflächen”, Acta Math. 105 (1961), 245-375. MR 25 \#4519a Zbl 0100.19402

[Jaco and Oertel 1984] W. Jaco and U. Oertel, "An algorithm to decide if a 3-manifold is a Haken manifold”, Topology 23:2 (1984), 195-209. MR 85j:57014 Zbl 0545.57003

[Jaco and Rubinstein 2003] W. Jaco and J. H. Rubinstein, "0-efficient triangulations of 3-manifolds", J. Differential Geom. 65:1 (2003), 61-168. MR 2005d:57034 Zbl 1068.57023

[Matveev 2003] S. Matveev, Algorithmic topology and classification of 3-manifolds, Algorithms and Computation in Mathematics 9, Springer, Berlin, 2003. MR 2004i:57026 Zbl 1048.57001

[Schleimer 2001] S. Schleimer, Almost normal Heegaard splittings, $\mathrm{PhD}$ thesis, University of California, Berkeley, CA, 2001.

[Thurston 1986] W. P. Thurston, "A norm for the homology of 3-manifolds", Mem. Amer. Math. Soc. 59:339 (1986), i-vi and 99-130. MR 88h:57014 Zbl 0585.57006

[Tollefson 1995] J. L. Tollefson, "Isotopy classes of incompressible surfaces in irreducible 3-manifolds”, Osaka J. Math. 32:4 (1995), 1087-1111. MR 97f:57023 Zbl 1004.57501

[Tollefson and Wang 1996] J. L. Tollefson and N. Wang, "Taut normal surfaces", Topology 35:1 (1996), 55-75. MR 97j:57022 Zbl 0868.57022

[Ziegler 1995] G. M. Ziegler, Lectures on polytopes, Graduate Texts in Mathematics 152, Springer, New York, 1995. MR 96a:52011 Zbl 0823.52002

Received October 23, 2007. Revised August 12, 2008.

DARYL COOPER

DEPARTMENT OF MATHEMATICS

UNIVERSITY OF CALIFORNIA

SANTA BARBARA, CA 93106

UNITED STATES

cooper@math.ucsb.edu

STEPHAN TILLMANN

DEPARTMENT OF MATHEMATICS AND STATISTICS

The University of MElbourne

Melbourne VIC 3010

Australia

tillmann@ms.unimelb.edu.au 